Research Article

\title{
Some Applications of Supra Preopen Sets
}

\author{
M. E. El-Shafei, ${ }^{1}$ A. H. Zakari, $^{2}$ and T. M. Al-shami ${ }^{3}{ }^{3}$ \\ ${ }^{1}$ Department of Mathematics, Mansoura University, Mansoura, Egypt \\ ${ }^{2}$ Department of Mathematics, Jazan University, Jazan, Saudi Arabia \\ ${ }^{3}$ Department of Mathematics, Sana'a University, Sana'a, Yemen
}

Correspondence should be addressed to T. M. Al-shami; tareqalshami83@gmail.com

Received 29 March 2020; Accepted 7 May 2020; Published 20 May 2020

Academic Editor: Ljubisa Kocinac

Copyright ( 2020 M. E. El-Shafei et al. This is an open access article distributed under the Creative Commons Attribution License, which permits unrestricted use, distribution, and reproduction in any medium, provided the original work is properly cited.

\begin{abstract}
The aim of this work is to define some concepts on supra topological spaces using supra preopen sets and investigate main properties. We started this paper by correcting some results obtained in previous study and presenting further properties of supra preopen sets. Then, we introduce a concept of supra prehomeomorphism maps and discuss its main properties. After that we explore the concepts of supra limit and supra boundary points of a set with respect to supra preopen sets and examine their behaviours on the spaces that possess the difference property. Finally, we formulate the concepts of supra pre- $T_{i}$-spaces $(i=0,1,2,3,4)$ and give completely descriptions for each one of them. In general, we study their main properties in detail and show the implications of these separation axioms among themselves as well as with $S T_{i}$-space with the help of some interesting examples.
\end{abstract}

\section{Introduction and Preliminaries}

A set $X$ with a family $\mu$ of its subsets is called a supra topological space [1], denoted by $(X, \mu)$, if $X \in \mu$ and the arbitrary union of members of $\mu$ is in $\mu$. Mashhour et al. [1] generalized some topological notions such as interior and closure operators and continuity and separation axioms. AlShami [2] has studied the classical topological notions such as limit points of a set, compactness, and separation axioms on the supra topological spaces.

Some results via topology are not still valid via supra topology such as the distribution of the closure operator between the union of two sets and the distribution of the interior operator between the intersection of two sets. Also, the property of a compact subset of a $T_{2}$-space is closed and is invalid on the supra topologies. To extend a class of supra open sets, the notions of supra $\alpha$-open [3], supra preopen [4], supra $b$-open [5], supra $\beta$-open [6], supra $R$-open [7], and supra semiopen sets [8] have been introduced and their main properties have been discussed. These generalizations of supra open sets were defined in a similar way of defining them on general topology. In other words, their definitions were formulated using supra interior and supra closure operators instead of interior and closure operators. These generalizations have been utilized to define new versions of compactness and connectedness, see, for example [9-14]. Mustafa and Qoqazeh [15] took advantage of supra $D$-sets to define separation axioms on supra topological spaces. Recently, Al-Shami and El-Shafei [16] have studied separation axioms on supra soft topological spaces.

It should be noted that the supra topological frame can be more convenient to solve some practical problems and to model some phenomena as pointed out in [17]. Also, the possibility of applying semiopen sets to deal with some problems on digital topology has been demonstrated in [18].

The layout of the paper is as follows. In Section 2, we correct some results of [4] and investigate further properties of supra preopen sets. Also, it presents the concept of supra prehomeomorphism maps and explores main properties. The concepts of supra prelimit and supra preboundary points of a set are studied in Section 3. Section 4 introduces new types of separation axioms using supra preopen sets and elucidates the relationships between them with the help of examples. Section 5 concludes the paper with summary and further works. 
In what follows, we collect the relevant definitions and results from supra topology and supra preopen sets to make this paper self-contained and easy to read.

Definition 1 (see [1]). A family $\mu$ of subsets of a nonempty set $X$ is called a supra topology provided that the following two conditions hold:

(1) $X$ and $\varnothing \in \mu$

(2) $\mu$ is closed under arbitrary union

Then, the pair $(X, \mu)$ is called a supra topological space. Every element of $\mu$ is called a supra open set and its complement is called a supra closed set.

\section{Remark 1}

(1) Since $\cup_{i \in \varnothing} G_{i}=\varnothing$, then some authors remove the empty set $\varnothing$ from the first condition of a supra topology

(2) $\mu$ is called an associated supra topology with a topology $\tau$ if $\tau \subseteq \mu$

(3) Through this paper, we consider $(X, \mu)$ and $(Y, \nu)$ are associated supra topological spaces with the topological spaces $(X, \tau)$ and $(Y, \theta)$, respectively

Definition 2 (see [1]). Let $A$ be a subset of $(X, \mu)$. Then, int $^{\mu}(A)$ is the union of all supra open sets contained in $A$ and $c l^{\mu}(A)$ is the intersection of all supra closed sets containing A.

If there is no confusion, we write int $(A)$ and $c l(A)$ in the places of $\operatorname{int}^{\mu}(A)$ and $c l^{\mu}(A)$, respectively.

Definition 3 A subset $A$ of $(X, \mu)$ is said to be

(1) Supra $\alpha$-open [3] if $A \subseteq \operatorname{int}(\operatorname{cl}(\operatorname{int}(A)))$

(2) Supra preopen [4] if $A \subseteq \operatorname{int}(\operatorname{cl}(A))$

(3) Supra $b$-open [5] if $A \subseteq \operatorname{int}(c l(A)) \cup c l(\operatorname{int}(A))$

Definition 4 (see [1]). For a subset $A$ of $(X, \mu), \operatorname{pint}^{\mu}(A)$ is the union of all supra preopen sets contained in $A$ and $p c l^{\mu}(A)$ is the intersection of all supra preclosed sets containing $A$.

If there is no confusion, we write $\operatorname{pint}(A)$ and $\operatorname{pcl}(A)$ in the places of $\operatorname{pint}^{\mu}(A)$ and $p c l^{\mu}(A)$, respectively.

Definition 5 (see [4]). A map $g:(X, \mu) \longrightarrow(Y, \nu)$ is said to be

(1) Supra precontinuous if $g^{-1}(H)$ is a supra preopen set in $X$ for each open set $H$ in $Y$

(2) Supra preopen (resp. supra preclosed) if $g(H)$ is a supra preopen (supra preclosed) set in $Y$ for each open (resp. closed) set in $X$

Theorem 1 (see [4]). For a map $g:(X, \mu) \longrightarrow(Y, \nu)$, we have the following results for every $A \in X$
(1) $g$ is supra precontinuous if and only if $g\left(p c l^{\mu}\right.$ $(A)) \subseteq c l^{\theta}(g(A))$

(2) $g$ is supra preopen if and only if $g\left(\right.$ int $\left.^{\tau}(A)\right) \subseteq$ $\operatorname{pint}^{\nu}(g(c l(A)))$

(3) $g$ is supra preclosed if and only if $\operatorname{pcl}^{\nu}(g(A)) \subseteq$ $g\left(c l^{\tau}(A)\right)$

Definition 6 (see [1]). A map $g:(X, \mu) \longrightarrow(Y, \nu)$ is said to be

(1) $S^{\star}$-continuous if $g^{-1}(H)$ is a supra open set in $X$ for every supra open set $H$ in $Y$

(2) $S^{\star}$-open (resp. $S^{\star}$-closed) if $g(H)$ is a supra open (resp. supra closed) set in $Y$ for every supra open set $H$ in $X$

(3) $S^{\star}$-homeomorphism if it is bijective, $S^{\star}$-continuous, and $S^{\star}$-open

Theorem 2. For a map $g:(X, \mu) \longrightarrow(Y, \nu)$, we have the following results for every $A \subseteq X$ :

(1) $g$ is $S^{\star}$-continuous if and only if $g\left(c l^{\mu}(A)\right) \subseteq c l^{\nu}(g(A))$

(2) $g$ is $S^{\star}$-open if and only if $g\left(\operatorname{int}^{\mu}(A)\right) \subseteq \operatorname{int}^{\nu}(g(c l(A)))$

(3) $g$ is $S^{\star}$-closed if and only if $c l^{\nu}(g(A)) \subseteq g\left(c l^{\mu}(A)\right)$

Definition 7 (see [1]). Let $A$ be a subset of $(X, \mu)$. The family $\mu_{A}=\{A \cap G: G \in \mu\}$ is called a supra relative topology on $A$. A pair $\left(A, \mu_{A}\right)$ is called a supra subspace of $(X, \mu)$.

Definition 8 (see [11]). $\beta$ is called a basis for a supra topology $(X, \mu)$ if every member of $\mu$ can be expressed as a union of elements of $\beta$.

Definition 9 (see [11]). Let $\left\{\left(X_{i}, \mu_{i}\right): i=1,2, \ldots, n\right\}$ be the collection of supra topological spaces. Then, $\beta=\prod_{i=1}^{n} \mu_{i}=$ $\left\{\prod_{i=1}^{n} G_{i}: G_{i} \in \mu_{i}\right\}$ defines a basis for a supra topology $T$ on $X=\prod_{i=1}^{n} X_{i}$. The pair $(X, T)$ is called a finite product supra spaces.

Theorem 3 (see [11]). A and B are supra preopen sets iff the product of them is supra preopen.

\section{Some Corrections and New Results of Supra Preopen Sets}

We begin this section with Proposition 1 below, originally proposed as Proposition 2.1 in [4].

\section{Proposition 1}

(1) The intersection of supra open and supra preopen is supra preopen

(2) The intersection of supra $\alpha$-open and supra preopen is supra preopen 
We give the below example to demonstrate that the above proposition need not be true in general.

Example 1. Let $\mu=\{\varnothing, X,\{1,4\},\{1,2,3\},\{2,3,4\}\}$ be a supra topology on $X=\{1,2,3,4\}$. Since $\operatorname{int}(c l(\{2,4\}))=X$, then $\{2,4\}$ is a supra preopen set and since int(cl(int $((\{1,3,4\}))))=X$, then $\{1,3,4\}$ is a supra $\alpha$-open set. Now, $\{1,4\} \cap\{2,4\}=\{4\}$ and $\{1,3,4\} \cap\{2,4\}=\{4\}$. Since int $(c l$ $(\{4\}))=\varnothing$ and $\operatorname{int}(c l(\operatorname{int}(\{4\})))=\varnothing$, then $\{4\}$ is neither a supra preopen set nor a supra $\alpha$-open set. This emphasizes that the above proposition is erroneous.

The above proposition is true in the case of $(X, \mu)$ is a topological space because the following two properties are satisfied on topological spaces, but they do not valid in supra topological spaces:

(1) $\operatorname{int}(A \cap B)=\operatorname{int}(A) \cap \operatorname{int}(B)$ for every subsets $A, B$ of a topological space $(X, \tau)$

(2) If $A$ is an open set, then $\operatorname{cl}(A) \cap B \subseteq \operatorname{cl}(A \cap B)$ for every subset $B$ of a topological space $(X, \tau)$

Proposition 2. Let $A$ be a supra b-open subset of $(X, \mu)$ such that $\operatorname{int}(A)=\varnothing$. Then, $A$ and $A^{c}$ are supra preopen.

Proof. Since $A$ is a supra $b$-open set, then $A \subseteq \operatorname{int}(c l$ $(A)) \cup c l(\operatorname{int}(A))$. Since $\operatorname{int}(A)=\varnothing$, then $\operatorname{cl}(\operatorname{int}(A))=\varnothing$. Therefore, $A \subseteq \operatorname{int}(c l(A))$. Thus, $A$ is a supra preopen set. Also, $(\operatorname{int}(A))^{c}=X$. This implies that $c l\left(A^{c}\right)=X$. Therefore, $A^{c}$ is supra preopen set.

In general, there does not exist a relationship between supra preopen sets in $(X, \mu)$ and its subspaces as the next two example show.

Example 2. Let $\mu=\{\varnothing, X,\{1,2,3\},\{1,2,4\}\}$ be a supra topology on $X=\{1,2,3,4\}$. If $Z=\{1,3\}$, then $\mu_{Z}=\{\varnothing, Z\}$ is a relative supra topology $Z$. In $(X, \mu)$, a set $B=\{3\}$ is not supra preopen. On the contrary, it can be seen that $B$ is a supra preopen set in $\left(Z, \mu_{Z}\right)$.

Theorem 4. The property of being a supra preopen set is preserved under $S^{\star}$-continuous and $S^{\star}$-open map.

Proof. Let a map $g:(X, \mu) \longrightarrow(Y, \nu)$ be $S^{\star}$-continuous and $S^{\star}$-open and let $A$ be a supra preopen subset of $(X, \mu)$. Obviously, $g(A) \subseteq g\left(\right.$ int $\left.^{\mu}\left(c l^{\mu}(A)\right)\right)$. Since $g$ is $S^{\star}$-open, then it follows from Theorem 2 that $g(A) \subseteq g\left(\right.$ int $\left.^{\mu}\left(c l^{\mu}(A)\right)\right) \subseteq$ $\operatorname{int}^{\nu}\left(g\left(c l^{\mu}(A)\right)\right)$. Since $g$ is $S^{\star}$-continuous, then it follows from Theorem 2 that $g(A) \subseteq$ int $^{\nu}\left(g\left(c l^{\mu}(A)\right)\right) \subseteq$ int $^{\nu}\left(c l^{\nu}\right.$ $(g(A)))$. Thus, $g(A) \subseteq \operatorname{int}^{\nu}\left(c l^{\nu}(g(A))\right)$. Hence, $g(A)$ is a supra preopen set.

Definition 10. For a nonempty subset $A$ of $(X, \mu)$, the family $\mu_{A}=\{A \cap G: G$ is a supra preopen subset of $(X, \mu)\}$ is called a relative pretopology on $A$. A pair $\left(A, \mu_{A}\right)$ is called a presubspace of $(X, \mu)$.
One can easily prove that a presubspace $\left(A, \mu_{A}\right)$ of $(X, \mu)$ is a supra topological space.

Proposition 3. Let $\left(Y, \mu_{Y}\right)$ be a presubspace of $(X, \mu)$. A subset $H$ of $Y$ is supra preclosed in $\left(Y, \mu_{Y}\right)$ iff there exists a supra preclosed subset $F$ of $(X, \mu)$ such that $H=Y \cap F$.

Proof

Necessity: let $H$ be a supra preclosed subset of $\left(Y, \mu_{Y}\right)$. Then, there exists a supra preopen subset $W$ of $\left(Y, \mu_{Y}\right)$ such that $H=Y \backslash W$. Now, there exists a supra preopen subset $V$ of $(X, \mu)$ such that $W=Y \cap V$. Therefore, $H=Y \backslash(Y \cap V)=Y \cap V^{c}$. By taking $F=V^{c}$, the proof of the necessary part is complete.

Sufficiency: let $H=Y \cap F$ such that $F$ is a supra preclosed subset of $(X, \mu)$. Then, $Y \backslash H=Y \backslash(Y \cap F)=$ $(Y \cap X) \backslash(Y \cap F)=Y \cap(X \backslash F)$. Since $X \backslash F$ is a supra preopen subset of $(X, \mu)$, then $Y \backslash H$ is a supra preopen subset of $\left(Y, \mu_{Y}\right)$. Thus, $H$ is a supra preclosed subset of $\left(Y, \mu_{Y}\right)$.

In the rest part of this section, we present a concept of supra prehomeomorphism and supra pre ${ }^{\star}$-homeomorphism maps and discuss some basic properties ( ${ }^{\star}$ denotes another type of homeomorphism maps).

Definition 11. A bijective map $g:(X, \mu) \longrightarrow(Y, \nu)$ is said to be a supra prehomeomorphism if it is supra precontinuous and supra preopen.

Since every supra open set is supra preopen, then every supra homeomorphism map is a supra prehomeomorphism. However, the converse is not always true as it is illustrated in the following example.

Example 3. Assume that $\tau=\{\varnothing, G \subseteq \mathbb{R}: 1 \in G\}$ and $\theta=\{\varnothing,\{1\}, \mathbb{R}\}$ are two topologies on the real numbers set $\mathbb{R}$. Let $\mu=\{\varnothing, G \subseteq \mathbb{R}: 1 \in \mathbb{R}$ or $2 \in \mathbb{R}\}$ and $\nu=\{\varnothing,\{1\},\{2,3\}$, $\{2,4\}, \mathbb{R}\}$ be two associated supra topologies with $\tau$ and $\theta$, respectively. Then, the identity map $g:(\mathbb{R}, \mu) \longrightarrow(\mathbb{R}, \nu)$ is a supra prehomeomorphism, but it is not a supra homeomorphism because the image of a supra open set $\{1,3\}$ is on a supra open set.

Theorem 5. The equivalence of the following properties hold if a map $g:(X, \mu) \longrightarrow(Y, \nu)$ is bijective and supra precontinuous:

(1) $g$ is a supra prehomeomorphism

(2) $g^{-1}$ is supra precontinuous

(3) $g$ is supra preclosed

Proof. Straightforward.

Theorem 6. A bijective map $g:(X, \mu) \longrightarrow(Y, \nu)$ is a supra prehomeomorphism if and only if $\mathrm{pcl}^{\nu}(g(A)) \subseteq g\left(\mathrm{cl}^{\tau}(A)\right)$ and $g\left(p c l^{\mu}(A)\right) \subseteq c l^{\theta}(g(A))$ for every $A \subseteq X$. 
Proof. ' $\Longrightarrow$ If $g$ is a supra prehomeomorphism map. Then, $g$ is supra precontinuous and supra preclosed. It follows from Theorem 1 that $p c l^{\nu}(g(A)) \subseteq g\left(c l^{\tau}(A)\right)$ and $g\left(p c l^{\mu}(A)\right) \subseteq c l^{\theta}(g(A))$.

' $\Longrightarrow$ ' If $g$ is a bijective map such that $p^{\nu} l^{\nu}(g$ $(A)) \subseteq g\left(c l^{\tau}(A)\right)$ and $g\left(p c l^{\mu}(A)\right) \subseteq c l^{\theta}(g(A))$, then $g$ is supra precontinuous and supra preclosed. It follows from Theorem 1 that $g$ is a supra prehomeomorphism map.

Definition 12. A map $g:(X, \mu) \longrightarrow(Y, \nu)$ is said to be

(1) Supra pre $e^{\star}$-continuous if $g^{-1}(H)$ is a supra preopen set in $X$ for every supra preopen set in $Y$

(2) Supra pre ${ }^{\star}$-open (resp. supra pre ${ }^{\star}$-closed) if $g(H)$ is a supra preopen (resp. supra preclosed) set in $Y$ for every supra preopen (resp. supra preclosed) set in $X$

(3) Supra pre ${ }^{\star}$-homeomorphism if it is bijective, supra pre $^{\star}$-continuous, and supra pre ${ }^{\star}$-open

\section{Limit and Boundary Points of a Set with Respect to Supra Preopen Sets}

This section defines the concepts of supra prelimit and supra preboundary points of a set and studies the interrelations between them. It provides some examples to show the obtained results and examines some properties of the supra prederived set on the spaces that possess the difference property.

Definition 13. A subset $A$ of $(X, \mu)$ is said to be a supra preneighbourhood of $x \in X$ provided that there is a supra preopen set $F$ containing $x$ such that $x \in F \subseteq A$.

Definition 14. A point $x \in X$ is said to be a supra prelimit point of a subset $A$ of $(X, \mu)$ provided that every supra preneighborhood of $x$ contains at least one point of $A$ other than $x$ itself.

All supra prelimit points of $A$ is said to be a supra prederived set of $A$ and is denoted by $A^{p^{\prime}}$.

Proposition 4. If $A \subseteq B$, then $A^{p^{\prime}} \subseteq B^{p^{\prime}}$ for every subsets $A$ and $B$ of $(X, \mu)$.

Proof. Straightforward.

Corollary 1. We have the following results for any two subsets $A$ and $B$ of $(X, \mu)$ :

(1) $A^{p^{\prime}} \cup B^{p^{\prime}} \subseteq(A \cup B)^{p^{\prime}}$

(2) $(A \cap B)^{p^{\prime}} \subseteq A^{p^{\prime}} \cap B^{p^{\prime}}$

The following example illustrates that the converse of the above proposition and corollary fails.

Example 4. Let $\mu=\{\varnothing, X,\{1,2\},\{1,3\},\{1,2,3\},\{1,2,4\}$, $\{1,3,4\},\{2,3,4\}\}$ be a supra topology on $X=\{1,2,3,4\}$. Then, $\{\varnothing, X,\{1,2\},\{1,3\},\{1,4\},\{2,3\},\{1,2,3\}, \quad\{1,2,4\}$, $\{1,3,4\},\{2,3,4\}\}$ is the collection of all supra preopen subsets of $(X, \mu)$. If $A=\{1,2,3\}$ and $B=\{1,4\}$, then $A^{p^{\prime}}=\{2,3,4\}$ and $B^{p^{\prime}}=\{4\}$. Obviously, $X^{p^{\prime}}=X$. Now, we have the following cases:

(i) $B^{p^{\prime}} \subseteq A^{p^{\prime}}$, but $B \subseteq A$

(ii) $A^{p^{\prime}} \cup B^{p^{\prime}}=\{2,3,4\}$ and $(A \cup B)^{p^{\prime}}=X^{p^{\prime}}=X$

(iii) $A^{p^{\prime}} \cap B^{p^{\prime}}=\{4\}$ and $(A \cap B)^{p^{\prime}}=\varnothing$

Proposition 5. Let $A$ be a subset of $(X, \mu)$ and $x \in X$. Then, $x \in A^{p^{\prime}}$ if and only if $x \in(A \backslash\{x\})^{p^{\prime}}$.

Proof

Necessity: let $x \in A^{p^{\prime}}$. Then, for every supra preopen set $G$ containing $x$, we have $(G \backslash\{x\}) \cap A \neq \varnothing$. Therefore, $(G \backslash\{x\}) \cap(A \backslash\{x\}) \neq \varnothing$. Thus, $x \in(A \backslash\{x\})^{p^{\prime}}$.

Sufficiency: it follows from Proposition 4.

Theorem 7. Let $A$ be a subset of $(X, \mu)$. Then, the following results hold.

(i) $A$ is a supra preclosed set iff $A^{p^{\prime}} \subseteq A$

(ii) $A \cup A^{p^{\prime}}$ is a supra preclosed set

(iii) $\operatorname{pcl}(A)=A \cup A^{p^{\prime}}$

Proof

(i) Suppose that $A$ is a supra preclosed set and $x \notin A$. Then, $A^{c}$ is a supra preopen set containing $x$. In this case, $A^{c} \cap A=\varnothing$ leads to $x \notin A^{p^{\prime}}$. Therefore, $A^{p^{\prime}} \subseteq A$. Conversely, let $x \in A^{c}$ and let $A^{p^{\prime}} \subseteq A$. Then, $x \notin A^{p^{\prime}}$. Therefore, there is a supra preopen set $G_{x}$ such that $G_{x} \backslash\{x\} \cap A=\varnothing$. Since $x \in A^{c}$, then $G_{x} \cap A=\varnothing$. Now, $G_{x} \subseteq A^{c}$. Therefore, $A^{c}=\cup\left\{G_{x}: x \in A^{c}\right\}$. Thus, $A$ is supra preclosed.

(ii) Let $x \notin\left(A \cup A^{p^{\prime}}\right)$. Then, $x \notin A$ and $x \notin A^{p^{\prime}}$. Therefore, there is a supra preopen set $G$ such that

$$
G \cap A=\varnothing \text {. }
$$

Now, for each $x \in G$, we have $x \notin A^{p^{\prime}}$. This means that

$$
G \cap A^{p^{\prime}}=\varnothing
$$

From (1) and (2), we obtain $G \cap\left(A \cup A^{p^{\prime}}\right)=\varnothing$. This implies that $x \notin\left(A \cup A^{p^{\prime}}\right)^{p^{\prime}}$. Hence, $\left(A \cup A^{p^{\prime}}\right)^{p^{\prime}} \subseteq\left(A \cup A^{p^{\prime}}\right)$. By (i), $A \cup A^{p^{\prime}}$ is a supra preclosed set, as required.

(iii) Since $A \subseteq p c l(A)$ and $A^{p^{\prime}} \subseteq(\operatorname{pcl}(A))^{p^{\prime}} \subseteq \operatorname{pcl}(A)$, then $A \cup A^{p^{\prime}} \subseteq \operatorname{pcl}(A)$. Since $A \cup A^{p}$ is a supra preclosed set containing $A$ and $\operatorname{pcl}(A)$ is the smallest supra preclosed set containing $A$, then $\operatorname{pcl}(A) \subseteq A \cup A^{p^{\prime}}$. Therefore, $\operatorname{pcl}(A)=A \cup A^{p^{\prime}}$. 
Corollary 2. If $A$ is a supra preclosed subset of $(X, \mu)$, then $A^{p^{\prime}},\left(A^{p^{\prime}}\right)^{p^{\prime}},\left(\left(A^{p^{\prime}}\right)^{p^{\prime}}\right)^{p^{\prime}}, \ldots$, are supra preclosed sets.

Theorem 8. If $g:(X, \mu) \longrightarrow(Y, \nu)$ is a supra pre $e^{\star}-h o$ meomorphism map, then $g\left(A^{p^{\prime}}\right)=(g(A))^{p^{\prime}}$ for each $A \subseteq X$.

Proof. Let $a \notin(g(A))^{p^{\prime}}$. Then, there is a supra preopen set $H$ containing $a$ such that $(H \backslash\{a\}) \cap g(A)=\varnothing$. So, $g^{-1}[(H \backslash\{a\}) \cap g(A)]=g^{-1}(\varnothing)$. This implies that $\left(g^{-1}(H) \backslash g^{-1}(a)\right) \cap A=\varnothing$. Thus, $g^{-1}(a) \notin A^{p}$. Since $g$ is bijective, then $a \notin g\left(A^{p^{\prime}}\right)$. Therefore, $g\left(A^{p^{\prime}}\right) \subseteq(g(A))^{p^{\prime}}$. By reversing the preceding steps, we find that $(g(A))^{p} \subseteq g\left(A^{p^{\prime}}\right)$. Hence, the proof is complete.

Definition 15. For a nonempty set $X$, a subcollection $\Lambda$ of $2^{X}$ is said to have the difference property provided that $G \in \Lambda$ implies that $G \backslash\{x\} \in \Lambda$.

The following two examples illustrate the existence and uniqueness of the difference property.

Example 5. Let $\mu=\{\varnothing, G \subseteq \mathbb{N}: G$ is infinite $\}$ be a supra topology on the set of natural numbers $\mathbb{N}$. It is clear that the infinity of $G$ implies the infinity of $G \backslash\{x\}$. That is, $G \in \mu$ implies $G \backslash\{x\} \in \mu$. Then, $(\mathbb{N}, \mu)$ has the difference property. Also, it can be seen that the collection of supra preopen subsets of $(\mathbb{N}, \mu)$ coincides with the collection of supra open sets. Hence, $(\mathbb{N}, \mu)$ has the difference property for the collection of supra preopen sets.

Example 6. Let $\mu=\{\varnothing, \mathbb{N} \backslash\{1,3\}, \mathbb{N} \backslash\{1,4\}, \mathbb{N} \backslash\{2,3\}, \mathbb{N} \backslash$ $\{2,4\}\} \cup\{G \subseteq \mathbb{N}$ : $G$ such that $\{1,2\} \subseteq G$ or $\{3,4\} \subseteq G\}$ be a supra topology on the set of natural numbers $\mathbb{N}$. Then, $\{1,2\} \in \mu$, but $\{1,2\} \backslash\{2\}=\{1\} \notin \mu$. Therefore, $(X, \mu)$ does not have the difference property for the collection of supra open sets. Hence, it does not have the difference property for the collection of supra preopen sets.

Theorem 9. If $(X, \mu)$ has the difference property for the collection of supra preopen sets, then the following properties hold for $A \subseteq X$ :

(1) $\left(A^{p^{\prime}}\right)^{p^{\prime}} \subseteq A^{p^{\prime}}$

(2) $\operatorname{pcl}\left(A^{p^{\prime}}\right)=A^{p^{\prime}}=(\operatorname{pcl}(A))^{p^{\prime}}$

(3) $A^{p^{\prime}}=\varnothing$ if $A$ is finite

Proof

(1) Let $x \notin A^{p^{\prime}}$. Then, there is a supra preopen set $G$ containing $x$ such that $G \backslash\{x\} \cap A=\varnothing$. Since $(X, \mu)$ has the difference property for the collection of supra preopen sets, then $G \backslash\{x\}$ is a supra preopen set. Therefore, $G \backslash\{x\} \cap A^{p^{\prime}}=\varnothing$. Since $x \notin A^{p^{\prime}}$, then $G \cap$ $\left(A^{p^{\prime}}\right)^{p^{\prime}}=\varnothing$. Thus, $x \notin\left(A^{p^{\prime}}\right)^{p^{\prime}}$. Hence, $\left(A^{p^{\prime}}\right)^{p^{\prime}} \subseteq A^{p^{\prime}}$.

(2) Since $\left(A^{p^{\prime}}\right)^{p^{\prime}} \subseteq A^{p^{\prime}}$, then it follows from Theorem 7 that $A^{p^{\prime}}$ is a supra preclosed set. Therefore,

$$
\operatorname{pcl}\left(A^{p^{\prime}}\right)=A^{p^{\prime}} .
$$

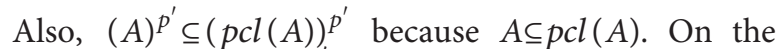
contrary, let $x \notin(A)^{p^{\prime}}$. Then, it follows from 1 above that $G\{x\} \cap A=\varnothing$ and $G \backslash\{x\} \cap A^{p^{\prime}}=\varnothing$. This means that $G \backslash\{x\} \cap p c l(A)=\varnothing$. Therefore, $x \notin(\operatorname{pcl}(A))^{p^{\prime}}$. Thus, $(\operatorname{pcl}(A))^{p^{\prime}} \subseteq(A)^{p^{\prime}}$. Hence,

$$
(\operatorname{pcl}(A))^{p^{\prime}}=(A)^{p^{\prime}} .
$$

From (3) and (4), the desired result is proved.

(3) Let $A$ be a finite subset of $X$. Suppose that there exists an element $x \in X$ such that $x \in A^{p^{\prime}}$. Then, for every supra preopen set $G$ containing $x$, we have $G \backslash\{x\} \cap A \neq \varnothing$. Therefore, for every $y \in A$ such that $y \neq x$, we have $G \backslash\{x, y\}$ is a supra preopen set. Thus, $G \backslash[A \cup\{x\}]$ is a supra preopen set such that $G \backslash[A \cup\{x\}] \cap A=\varnothing$. This implies that $x \notin A^{p^{\prime}}$. However, this is a contradiction. Hence, it must be that $A^{p^{\prime}}=\varnothing$.

We explain that the three properties mentioned in the above theorem need not be true if $(X, \mu)$ does not have the difference property for the collection of supra preopen sets. Let $A=\{2,4,5\}$ be a subset of supra topological space given in Example 6. Note that the collection of supra open sets coincides with the collection of supra preopen sets. By calculating, we find that $A^{p^{\prime}}=\mathbb{N} \backslash\{2,4\},\left(A^{p^{\prime}}\right)^{p^{\prime}}=\mathbb{N} \backslash\{1,3\}$, and $\operatorname{cl}\left(A^{p}\right)=\mathbb{N}$. This leads to the following three properties:

(1) $\left(A^{p^{\prime}}\right)^{p^{\prime}} \subseteq A^{p^{\prime}}$

(2) $\operatorname{pcl}\left(A^{p^{\prime}}\right) \neq A^{p^{\prime}}$

(3) $A^{p^{\prime}} \neq \varnothing$ in spite of $A$ is finite

Definition 16. Let $A$ be a subset of $(X, \mu)$. The supra preboundary of $A$ (denoted by $p b(A)$ ) is the set of all elements which belongs to $\left(p \operatorname{pint}(A) \cup \operatorname{pint}\left(A^{c}\right)\right)^{c}$.

Lemma 1. Let $A$ be a subset of $(X, \mu)$. Then,

(1) $(\operatorname{pint}(A))^{c}=\operatorname{pcl}\left(A^{c}\right)$

(2) $(\operatorname{pcl}(A))^{c}=\operatorname{pint}\left(A^{c}\right)$

Proof. We prove (1) and (2) is proved in a similar way. Since pint $(A) \subseteq A$, then $A^{c} \subseteq(\operatorname{pint}(A))^{c}$. Therefore, $p c l$ $\left(A^{c}\right) \subseteq \operatorname{pcl}\left(\operatorname{pint}(A)^{c}\right)=(\operatorname{pint}(A))^{c}$. Conversely, $A^{c} \subseteq \operatorname{pcl}\left(A^{c}\right)$. Then, $\quad\left(\operatorname{pcl}\left(A^{c}\right)\right)^{c} \subseteq A$. Therefore, $\quad \operatorname{pint}\left(\left(\operatorname{pcl}\left(A^{c}\right)\right)^{c}\right)=$ $\left(\operatorname{pcl}\left(A^{c}\right)\right)^{c} \subseteq \operatorname{pint}(A)$. Thus, $(\operatorname{pint}(A))^{c} \subseteq \operatorname{pcl}\left(A^{c}\right)$. Hence, the desired result is proved.

Proposition 6. Let $A$ be a subset of $(X, \mu)$. Then, $p b(A)=\operatorname{pcl}(A) \cap \operatorname{pcl}\left(A^{c}\right)$.

Proof. $p b(A)=\left\{x \in X: x \notin \operatorname{pint}(A)\right.$ and $\left.x \notin p \operatorname{pint}\left(A^{c}\right)\right\}$

$$
=\left\{x \in X: x \notin\left(\operatorname{pcl}\left(A^{c}\right)\right)^{c} \text { and } x \notin(\operatorname{pcl}(A))^{c}\right\}
$$


$=\left\{x \in X: x \in \operatorname{pcl}\left(A^{c}\right)\right.$ and $\left.\quad x \in \operatorname{pcl}(A)\right\}=\operatorname{pcl}(A) \cap$ $\operatorname{pcl}\left(A^{c}\right)$.

Corollary 3. Let $A$ be a subset of $(X, \mu)$. Then,
(1) $p b(A)=p b\left(A^{c}\right)$
(2) $p b(A)$ is a supra preclosed set
(3) $p b(A)=\operatorname{pcl}(A) \backslash \operatorname{pint}(A)$
(4) $\operatorname{pcl}(A)=\operatorname{pint}(A) \cup p b(A)$
(5) $\operatorname{pcl}(A)=A \cup p b(A)$
(6) $\operatorname{pint}(A)=A \backslash p b(A)$
(7) $p b(p c l(A)) \subseteq p b(A)$

Proof

(1) $\operatorname{pb}(A)=\operatorname{pcl}(A) \cap \operatorname{pcl}\left(A^{c}\right)=\operatorname{pcl}\left(\left(A^{c}\right)^{c}\right) \cap \operatorname{pcl}\left(A^{c}\right)=$ $p b\left(A^{c}\right)$

(2) It follows from noting that $p b(A)$ is the intersection of two supra preclosed sets which is supra preclosed

(3) $\operatorname{pb}(A)=\operatorname{pcl}(A) \cap \operatorname{pcl}\left(A^{c}\right)=\operatorname{pcl}(A) \cdot \cap(\operatorname{pint}(A))^{c}=$ $\operatorname{pcl}(A) \backslash \operatorname{pint}(A)$

(4) $\operatorname{pint}(A) \cup p b(A)=\operatorname{pint}(A) \cup(p c l(A) \backslash p \operatorname{int}(A))=p$ $\operatorname{cl}(A)$

(5) $A \cup p b(A)=A \cup(p c l(A) \backslash p \operatorname{pint}(A))=\operatorname{pcl}(A)$

(6) $A \backslash p b(A)=A \cap(p c l(A) \backslash p \operatorname{int}(A))^{c}=A \cap(p c l(A))^{c}$ $\cup(\operatorname{pint}(A))=\operatorname{pint}(A)$

(7) $p b(p c l(A))=p c l(p c l(A)) \cap p c l \quad\left((p c l(A))^{c}\right)=p c l$ $(A) \cap \operatorname{pcl}\left((\operatorname{pcl}(A))^{c}\right)$; Since $(\operatorname{pcl}(A))^{c} \subseteq A^{c}$, then the result holds

Proposition 7. Let $A$ be a subset of $(X, \mu)$. Then,

(1) $A$ is a supra preopen set iff $p b(A) \cap A=\varnothing$

(2) $A$ is a supra preclosed set iff $p b(A) \subseteq A$

Proof.

(1) Necessity: since $A$ is supra preopen, then $p b(A) \cap A=p b(A) \cap \operatorname{pint}(A)=\varnothing$.

Sufficiency: let $x \in A$. Then, $x \in \operatorname{pint}(A)$ or $x \in p b(A)$. Since $p b(A) \cap A=\varnothing$, then $x \in \operatorname{pint}(A)$. Therefore, $A \subseteq \operatorname{pint}(A)$. Thus, $A$ is supra preopen.

(2) Necessity: since $A$ is supra preclosed, then $p b(A)=p c l(A) \backslash \operatorname{pint}(A)=A \backslash \operatorname{pint}(A)$. Therefore, $p b(A) \subseteq A$.

Sufficiency: suppose that $p b(A) \subseteq A$ and let $x \in A^{c}$. Then, $\quad x \notin p b(A)=\operatorname{pcl}(A) \backslash \operatorname{pint}(A)$. Since $x \notin \operatorname{pint}(A)$, then $x \notin \operatorname{pcl}(A)$ so that for each supra preopen set $G$ containing $x$, we obtain $G \cap A=\varnothing$. This implies that $x \in G \subseteq A^{c}$. Thus, $A^{c}$ is a union of supra preopen sets. Hence, $A^{c}$ is supra preopen.
Corollary 4. We have the following properties for a subset $A$ of $(X, \mu)$ :

(1) $A$ is both supra preopen and supra preclosed iff $p b(A)=\varnothing$

(2) $p b(p \operatorname{int}(A))=\varnothing$

(3) $\operatorname{pint}(p b(A))=\varnothing$

\section{Separation Axioms with Respect to Supra Preopen Sets}

In this section, we use a class of supra preopen sets to introduce new types of separation axioms, namely, $S p T_{i}$-spaces $(i=0,1,2,3,4)$. We establish some characterizations of each one of these spaces and study them in terms of hereditary and topological properties and product space. Illustrative examples are provided to show the obtained results.

Definition 17. A supra topological space $(X, \mu)$ is said to be

(1) $S p T_{0}$ if for every $a \neq b \in X$, there exists a supra preopen set containing only one of them.

(2) $S p T_{1}$ if for every $a \neq b \in X$, there exist two supra preopen sets one of them contains $a$ but not $b$ and the other contains $b$ but not $a$

(3) Supra pre-Hausdorff (or $S p T_{2}$ ) if for every $a \neq b \in X$, there exist two disjoint supra preopen sets $U$ and $V$ containing $a$ and $b$, respectively

(4) Supra preregular if for every supra preclosed set $F$ and each $a \notin F$, there exist disjoint supra preopen sets $U$ and $V$ containing $F$ and $a$, respectively

(5) Supra prenormal if for every disjoint supra preclosed sets $F$ and $H$, there exist disjoint supra preopen sets $U$ and $V$ containing $F$ and $H$, respectively

(6) $S p T_{3}$ (resp. $S p T_{4}$ ) if it is both supra preregular (resp. supra prenormal) and $S p T_{1}$

Theorem 10. The following three statements are equivalent:

(1) $(X, \mu)$ is an $S p T_{0}$-space

(2) $\operatorname{pcl}(\{a\}) \neq \operatorname{pcl}(\{b\})$ for each $a \neq b \in X$

(3) For each $a \in X$, we have $\{a\}^{p^{\prime}}$ is a union of supra preclosed sets

Proof. $1 \longrightarrow 2$ : for each $a \neq b \in X$, there exists a supra preopen set $G$ containing $a$ but not $b$ or containing $b$ but not $a$. Say $a \in G$ and $b \notin G$. Then, $a \notin p c l(\{b\})$ because $G$ is a supra preopen set containing $a$ such that $G \cap\{b\}=\varnothing$. Since $a \in \operatorname{pcl}(\{a\})$, then $\operatorname{pcl}(\{a\}), \neq \operatorname{pcl}(\{b\})$.

$2 \longrightarrow 3$ : let $b \in\{a\}^{p^{\prime}}$. Then, $b \neq a$ and $b \in\{a\} \cup$ $\{a\}^{p^{\prime}}=\operatorname{pcl}(\{a\})$. Therefore, $\operatorname{pcl}(b) \subseteq \operatorname{pcl}(\{a\})$. Thus, $b \in p c l$ $(b) \subseteq\{a\}^{p^{\prime}}$. Hence, $\{a\}^{p^{\prime}}=\cup\left\{p c l(b)\right.$ : for each $\left.b \in\{a\}^{p^{\prime}}\right\}$.

$3 \longrightarrow 1$ : let $a \neq b$. Then, we have two cases:

(i) Either $b \in\{a\}^{p^{\prime}}$. Then, there is a supra preclosed set $F$ such that $b \in F \subseteq\{a\}^{p^{\prime}}$. Since $a \notin\{a\}^{p^{\prime}}$, then $a \notin F$. 
Therefore, $F^{c}$ is a supra preopen set containing $a$ such that $b \notin F^{c}$.

(ii) Or $b \notin\{a\}^{p^{\prime}}$. Then, there is a supra preopen set $G$ containing $b$ such that $a \notin G$.

In the both cases above, we infer that $(X, \mu)$ is an $S p T_{0}$-space.

Corollary 5. An $S p T_{0}$-space $(X, \mu)$ contains at most a supra predense singleton set $(\{a\}$ is a supra predense set if $\operatorname{pcl}\{a\}=X)$.

Proof. Let $(X, \mu)$ be an $S p T_{0}$-space. Suppose that there are two distinct singleton set $\{a\}$ and $\{b\}$ such that $\operatorname{pcl}(\{a\})=\operatorname{pcl}(\{b\})=X$. Then, $(X, \mu)$ is not an $S p T_{0}$-space, a contradiction. Hence, $(X, \mu)$ contains at most a supra predense singleton set.

Theorem 11. The following there statements are equivalent:

(1) $(X, \mu)$ is an $S p T_{1}$-space

(2) Every singleton subset of $(X, \mu)$ is supra preclosed

(3) The intersection of all supra preopen sets containing a set $A$ is exactly $A$

(4) $\{a\}^{p^{\prime}}=\varnothing$ for each $a \in X$

Proof. $1 \longrightarrow 2$ : consider $(X, \mu)$ is an $S p T_{1}$-space and let $\{a\} \subseteq X$. For all $b \in X$ such that $a \neq b$, there exists a supra preopen set $G$ containing $b$ such that $G \cap\{a\}=\varnothing$. Then, $b \notin \operatorname{pcl}(\{a\})$. Therefore, $\operatorname{pcl}(\{a\})=\{a\}$. Thus, $\{a\}$ is a supra preclosed set.

$2 \longrightarrow 3$ : let $A$ be a subset of $(X, \mu)$. Then, for each $a \in A^{c}$, we have $\{a\}^{c}$ is a supra preopen set containing $A$. Now, $A \subseteq\{G: G$ is a supra preopen set containing $A\} \subseteq\left\{\{a\}^{c}: a \in A^{c}\right\} \subseteq A$. Thus, $A=\{G: G$ is a supra preopen set containing $A\}$, as required.

$3 \longrightarrow 4:$ suppose that there exists $a \in X$ such that $\{a\}^{p^{\prime}} \neq \varnothing$. Then, there exists $b \neq a$ such that $b \in\{a\}^{p^{\prime}}$. Therefore, $G \backslash\{b\} \cap\{a\} \neq \varnothing$ for every supra preopen set $G$ containing $b$. This implies that any supra preopen set containing $b$ contains $a$ as well. Thus, the intersection of all supra preopen sets containing $b$ is not equal $\{b\}$. However, this contradicts 3 . Hence, it must be $\{a\}^{p^{\prime}}=\varnothing$.

$4 \longrightarrow 1$ : let $a \neq b$. Since $\{a\}^{p^{\prime}}=\varnothing$ and $\{b\}^{p^{\prime}}=\varnothing$, then $\{a\}$ and $\{b\}$ are supra preclosed sets. Therefore, $\{a\}^{c}$ and $\{b\}^{c}$ are supra preopen sets containing $\{b\}$ and $\{a\}$, respectively. Thus, $(X, \mu)$ is an $S p T_{1}$-space.

Proposition 8. Every $(X, \mu)$ satisfying the difference property for the collection of supra preopen sets is an $S p T_{1}$-space.

Proof. Let $a \neq b \in X$. Since $X$ is a supra preopen set and $(X, \mu)$ satisfies the difference property for the collection of supra preopen sets, then $X \backslash\{a\}$ and $X \backslash\{b\}$ are supra preopen sets containing $b$ and $a$, respectively, such that $a \notin X \backslash\{a\}$ and $b \notin X \backslash\{b\}$. Hence, $(X, \mu)$ is an $S p T_{1}$-space.
We show by the following example that the converse of the above proposition is not always true.

Example 7. Let $\mu=\{\varnothing, X,\{1,2\},\{1,3\}\}$ be a supra topology on $X=\{1,2,3\}$. Then, the collection of all supra preopen subsets of $(X, \mu)$ is $\{\varnothing, X,\{1\},\{1,2\},\{1,3\},\{2.3\}\}$. Therefore, $(X, \mu)$ is an $S p T_{1}$-space. On the contrary, $(X, \mu)$ does not satisfy the difference property for the collection of supra preopen sets because $\{2,3\}$ is a supra preopen set, but $\{2,3\} \backslash\{3\}$ is not supra preopen.

We need the following definition to obtain the equivalence between $S p T_{0}$ and $S p T_{1}$.

Definition 18. $(X, \mu)$ is called a supra presymmetric space if $a \in \operatorname{pcl}\{b\}$ implies that $b \in \operatorname{pcl}\{a\}$ for $a \neq b \in X$.

Theorem 12. Let $(X, \mu)$ be a supra presymmetric space. Then, it is $S p T_{1}$ iff it is $S p T_{0}$.

Proof. The necessary condition is obvious.

To prove the sufficient condition, let $a \neq b$. Then, there exists a supra preopen set $G$ containing only one of them. Say, $a \in G$ and $b \notin G$. Therefore, $a \notin p c l\{b\}$. By the supra presymmetry of $(X, \mu)$, we have $b \notin p c l\{a\}$. Thus, $(p c l\{a\})^{c}$ is a supra preopen set containing $b$. Hence, $(X, \mu)$ is $S p T_{1}$.

Theorem 13. The following three statements are equivalent:

(1) $(X, \mu)$ is an $S p T_{2}$-space

(2) $\{a\}=\cap\left\{F_{i}: F_{i}\right.$ is a supra preclosed neighborhood of $\left.a\right\}$ for each $a \in X$

(3) The diagonal $\Delta=\{(a, a): a \in X\}$ is supra preclosed in the product supra space $X \times X$

Proof. $1 \longrightarrow 2$ : consider $(X, \mu)$ is an $S p T_{2}$-space. Then, for $a \neq b$, there exist two disjoint supra preopen sets $G_{i}$ and $H_{i}$ such that $a \in G_{i}$ and $b \in H_{i}$. Obviously, $G_{i} \subseteq H_{i}^{c}$. Therefore, $a \in \operatorname{pcl}\left(G_{i}\right) \subseteq H_{i}^{c}=F_{i}$. Thus, $F_{i}$ is a supra preclosed neighborhood of $a$ such that $b \notin F_{i}$. Hence, $\{a\}=\cap\left\{F_{i}: F_{i}\right.$ is a supra preclosed neighborhood of $a$ \}.

$2 \longrightarrow 1$ : to prove that $(X, \mu)$ is an $S p T_{2}$-space, let $a \neq b$. Since $\{a\}=\cap\left\{F_{i}: F_{i}\right.$ is a supra preclosed neighborhood of $\left.a\right\}$, then there exists a supra preclosed neighborhood $F_{i_{0}}$ of $a$ such that $b \notin F_{i_{0}}$. Therefore, there exists a supra preopen set $G$ containing $a$ such that $a \in \operatorname{pcl}(G) \subseteq F_{i_{0}}$. It is clear that $(\operatorname{pcl}(G))^{c}$ is a supra preopen set containing $b$ and $G \cap(p c l(G))^{c}=\varnothing$. Hence, $(X, \mu)$ is an $S p T_{2}$-space.

$1 \longrightarrow 3$ : suppose that $(X, \mu)$ is $S p T_{2}$ and let $(a, b) \in X \times X-\Delta$. Then, $a \neq b$. Therefore, there exist two disjoint supra preopen sets $G$ and $H$ containing $a$ and $b$, respectively. Thus, $(a, b) \in G \times H \subseteq X \times X-\Delta$, proving that $X \times X-\Delta$ is a supra preneighbourhood of any of its points. Hence, $\Delta$ is supra preclosed.

$3 \longrightarrow 1$ : suppose that $\Delta$ is a supra preclosed subset of $X \times X$ and let $a \neq b \in X$. Then, $X \times X-\Delta$ is a supra preopen set containing $(a, b)$. Therefore, there exist two supra preopen subsets $G$ and $H$ of $(X, \mu)$ such that 
$(a, b) \in G \times H \subseteq X \times X-\Delta$. This implies that $G$ and $H$ are two disjoint supra preopen sets containing $a$ and $b$, respectively. Hence, $(X, \mu)$ is $S p T_{2}$.

Theorem 14. The following three statements are equivalent:

(1) $(X, \mu)$ is supra preregular

(2) For each supra preopen subset $U$ of $(X, \mu)$ containing $a$, there exists a supra preopen subset $V$ of $(X, \mu)$ such that $a \in V \subseteq p c l(V) \subseteq U$

(3) Every supra preopen subset $U$ of $(X, \mu)$ can be represented as follows: $U=\cup\{H: H$ is a supra preopen subset of $(X, \mu)$ and $p c l(H) \subseteq U\}$

Proof. $1 \longrightarrow 2$ : let $(X, \mu)$ be a supra preregular space and $U$ be a supra preopen set such that $a \in U$. Then, there exist disjoint supra preopen sets $V$ and $W$ containing $a$ and $U^{c}$, respectively. Therefore, $a \in V \subseteq W^{c} \subseteq U$. Thus, $a \in V \subseteq$ $\operatorname{pcl}(V) \subseteq U$.

$2 \longrightarrow 3$ : suppose that $U$ is a supra preopen set. By hypothesis, for each $a \in U$, there exists a supra preopen set $H$ such that $a \in H \subseteq p c l(H) \subseteq U$. Then, $U=\cup\{H: H$ is supra preopen and $\operatorname{pcl}(H) \subseteq U\}$.

$3 \longrightarrow 1$ : let $F$ be a supra preclosed set such that $a \notin F$. Then, $F^{c}=\cup\left\{H: H\right.$ is supra preopen and $\left.p c l(H) \subseteq F^{c}\right\}$. Since $a \in F^{c}$, then there exists a supra preopen set $H_{a}$ containing $a$ such that $\operatorname{pcl}\left(H_{a}\right) \subseteq F^{c}$. Take $V=\left(\operatorname{pcl}\left(H_{a}\right)\right)^{c}$. Then, $V$ is a supra preopen set containing $F$ and $V \cap H_{a}=\varnothing$. This completes the proof.

Theorem 15. Consider $(X, \mu)$ is a supra preregular space. Then, the following concepts are equivalent:

(1) $(X, \mu)$ is an $S p T_{2}$-space

(2) $(X, \mu)$ is an $S p T_{1}$-space

(3) $(X, \mu)$ is an $S p T_{0}$-space

Proof. The implications $1 \longrightarrow 2 \longrightarrow 3$ are obvious.

$3 \longrightarrow 1$ : let $a, b \in X$ such that $a \neq b$. Since $(X, \mu)$ is an $S p T_{0}$-space, then from Theorem 10, we obtain $p c l\{a\} \neq p c l\{b\}$. Therefore, $a \notin p c l\{b\}$ or $b \notin p c l\{a\}$. Say, $a \notin p c l\{b\}$. Since $(X, \mu)$ is supra preregular, then there exist disjoint supra preopen sets $G$ and $H$ containing $a$ and $p c l\{b\}$, respectively. Thus, $(X, \mu)$ is an $S p T_{2}$-space.

Theorem 16. The following statements are equivalent:

(1) $(X, \mu)$ is supra prenormal

(2) For each supra preclosed set $F$ and each supra preopen set $U$ containing $F$, there exists a supra preopen set $V$ such that $F \subseteq V \subseteq p c l(V) \subseteq U$

(3) For every supra preopen sets $U$ and $V$ such that $U \cup V=X$, there are two supra preclosed sets $F$ and $H$ contained in $U$ and $V$, respectively, such that $F \cup H=X$
Proof. $1 \longrightarrow 2$ : consider $(X, \mu)$ is supra prenormal and $F$ is a supra preclosed subset of a supra preopen set $U$. Then, $U^{c}$ and $F$ are disjoint supra preclosed sets. Therefore, there exist two disjoint supra preopen sets $W$ and $V$ containing $U^{c}$ and $F$, respectively. Thus, $F \subseteq V \subseteq W^{c}=\operatorname{pcl}\left(W^{c}\right) \subseteq U$. Hence, $F \subseteq V \subseteq p c l(V) \subseteq U$.

$2 \longrightarrow 3$ : consider $U$ and $V$ are supra preopen sets such that $U \cup V=X$. Then, $U^{c}$ is a supra preclosed sets such that $U^{c} \subseteq V$. By 2, there is a supra preopen set $G$ such that $U^{c} \subseteq G \subseteq p c l(G) \subseteq V$. Thus, $G^{c} \subseteq U$ and $p c l(G) \subseteq V$ are supra preclosed sets such that $G^{c} \cup \operatorname{pcl}(G)=X$.

$3 \longrightarrow 1$ : consider $F$ and $H$ are disjoint supra preclosed sets. Since $F^{c}$ and $H^{c}$ are supra open sets such that $F^{c} \cup H^{c}=X$, then there are two supra preclosed sets $M$ and $N$ such that $M \subseteq F^{c}, N \subseteq H^{c}$, and $M \cup N=X$. Thus, $M^{c}$ and $N^{c}$ are two disjoint supra preopen sets containing $F$ and $H$, respectively. Hence, $(X, \mu)$ is supra prenormal.

Theorem 17. If all members of $\mu$ are supra clopen, then $(X, \mu)$ is an $S_{p} T_{4}$-space.

Proof. Let $a \in X$ such that $c l(\{a\})=A \subseteq X$. By hypothesis, $A$ is a supra clopen set. Then, int $(c l(\{a\}))=\operatorname{int}(A)=A$. Therefore, $\{a\}$ is a supra preopen set. The arbitrary selection of $x$ implies that every singleton subset of $X$ is supra preopen. Therefore, every subset of $X$ is supra preopen. Hence, the desired result is proved.

To explain that the converse of the above theorem is not always true, we provide the following example.

Example 8. Let $\mu=\{\varnothing, X,\{3\},\{1,2\},\{2,3\}\}$ be a supra topology on $X=\{1,2,3\}$. Then, the collection of all supra preopen subsets of $(X, \mu)$ is $\{\varnothing, X,\{2\},\{3\},\{1,2\}$, $\{1,3\},\{2,3\}\}$. It easily checks that $(X, \mu)$ is an $S p T_{4}$-space. On the contrary, a set $\{2,3\} \in \mu$ is not a supra clopen set.

Now, we show the implications of these separation axioms among themselves as well as with $S T_{i}$-space.

It should be noted that the concepts of $S T_{i}$-space which were defined by replacing "supra preopen" by "supra open" in Definition 4.1 , see $[1,2]$.

Theorem 18. Every $S p T_{i}$-space is $S p T_{i-1}$ for $i=1,2,3,4$.

Converse of this theorem is not necessary true as seen from the following examples.

Example 9. Let $\mu=\{\varnothing, X,\{4\},\{1,3,4\},\{2,3,4\},\{1,2,4\}\}$ be a supra topology on $X=\{1,2,3,4\}$. Then, the collection of all supra preopen subsets of $(X, \mu)$ is $\{\varnothing, X,\{4\},\{1,4\}$, $\{2,4\},\{3,4\},\{1,3,4\},\{2,3,4\},\{1,2,4\}\}$. Therefore, $(X, \mu)$ is not an $S p T_{1}$-space because $1 \neq 4$ and every supra preopen set containing 1 contains 4 as well. On the contrary, it can be checked that $(X, \mu)$ is $S p T_{0}$.

Example 10. Assume that $(X, \mu)$ is the same as in Example 7. Then, $(X, \mu)$ is not an $S p T_{2}$-space because $2 \neq 3$ and there do not exist disjoint supra preopen sets such that one of them 
contains 2 and the other contains 3. On the contrary, it can be checked that $(X, \mu)$ is $S p T_{1}$.

Example 11. Let $\mu=\{\varnothing, X,\{1,2\},\{3,4\},\{1,3\},\{2,4\},\{2,3\}$, $\{1,2,3\},\{1,2,4\},\{1,3,4\},\{2,3,4\}\}$ be a supra topology on $X=\{1,2,3,4\}$. In $(X, \mu)$, every set is supra open iff it is supra preopen. Now, $\{1,4\}$ is a supra preclosed set and $2 \notin\{1,4\}$. Since there do not exist two disjoint supra preopen sets such that one of them contains 2 and the other contains $\{1,4\}$, then $(X, \mu)$ is not supra $S p T_{3}$. On the contrary, it can be checked that $(X, \mu)$ is $S p T_{2}$.

Example 12. Let $\mu=\{\varnothing, X,\{2\},\{4\},\{2,4\},\{1,3\},\{1,4\},\{1,5\}$, $\{2,3\},\{2,5\},\{3,5\}, \quad\{4,5\},\{1,2,3\},\{1,2,4\},\{1,2,5\},\{1,3,4\}$, $\{1,3,5\},\{1,4,5\},\{2,3,4\},\{2,3,5\},\{2,4,5\},\{3,4,5\},\{1,2,3,4\}$, $\{1,2,3,5\},\{1,2,4,5\},\{1,3,4,5\},\{2,3,4,5\}\}$ be a supra topology on $X=\{1,2,3,4,5\}$. In $(X, \mu)$, every set is supra open iff it is supra preopen. Now, $\{1,2\}$ and $\{3,4\}$ are disjoint supra preclosed subsets of $(X, \mu)$. Since there do not exist two disjoint supra preopen sets such that one of them contains $\{1,2\}$ and the other contains $\{3,4\}$, then $(X, \mu)$ is not supra prenormal. Therefore, it is not $S p T_{4}$. On the contrary, one can check that $(X, \mu)$ is $S p T_{3}$.

Theorem 19. Every $S T_{i}$-space $(X, \mu)$ is $S p T_{i}$ for $i=0,1,2$.

Proof. It follows from the fact that every supra open set is supra preopen.

Converse of this theorem is not necessary true as is seen in the following examples.

Example 13. Let $\mu=\{\varnothing, X,\{1\},\{2,3\}\}$ be a supra topology on $X=\{1,2,3\}$. Then, $(X, \mu)$ is not an $S T_{0}$-space. On the contrary, it follows from Theorem 17 that $(X, \mu)$ is $S p T_{4}$.

We complete this section by discussing these separation axioms in terms of hereditary and topological properties and finite product space.

Definition 19. A property is said to be a relative prehereditary property if the property passes from a supra topological space to every relative presubspace.

Theorem 20. A property of being an $S p T_{i}$-space is a relative prehereditary for $i=0,1,2,3$.

Proof. We prove the theorem in the case of $i=3$ and the other cases follow similar lines.

Suppose that $\left(A, \mu_{A}\right)$ is a relative presubspace of an $\operatorname{SpT}_{3}$-space $(X, \mu)$. We first show that $\left(A, \mu_{A}\right)$ is an $S p T_{1}$-space. Let $x \neq y \in A \subseteq X$. Then, there are two supra preopen subsets $U$ and $V$ of $(X, \mu)$ containing $x$ and $y$, respectively, such that $x \notin V$ and $y \notin U$. Now, $G=A \cap U$ and $H=A \cap V$ are two supra preopen subsets of $\left(A, \mu_{A}\right)$ containing $x$ and $y$, respectively, such that $x \notin H$ and $y \notin G$. Thus, $\left(A, \mu_{A}\right)$ is $S p T_{1}$. Second, we show that $\left(A, \mu_{A}\right)$ is supra preregular. Let $H$ be a supra preclosed subset of $\left(A, \mu_{A}\right)$ and $a \in A$ such that $a \notin H$. It follows from Proposition 3 that there is a supra preclosed subset $F$ of $(X, \mu)$ such that $H=F \cap A$. Since $a \notin F$, then there exist disjoint supra preopen subsets $U$ and $V$ of $(X, \mu)$ containing $F$ and $a$, respectively. Now, $M=U \cap A$ and $N=V \cap A$ are disjoint supra preopen subsets of $\left(A, \mu_{A}\right)$ containing $H$ and $a$, respectively. Thus, $\left(A, \mu_{A}\right)$ is supra preregular. Hence, the proof is complete.

Proposition 9. Let $g:(X, \mu) \longrightarrow(Y, \theta)$ be an injective supra precontinuous map. If $(Y, \theta)$ is $T_{i}$, then $(X, \mu)$ is $S p T_{i}$ for $i=0,1,2$.

Proof. We only prove the proposition in the case of $i=2$ and the other cases can be made similarly.

Let $a \neq b \in X$. Then, it follows from the injectivity of $g$, that there are $x \neq y \in Y$ such that $x=f(a)$ and $y=f(b)$. Since $(Y, \theta)$ is $T_{2}$, then there are two disjoint open subsets $U$ and $V$ of $(Y, \theta)$ containing $a$ and $b$, respectively. Now, $g^{-1}(U)$ and $g^{-1}(V)$ are disjoint supra preopen subsets of $(X, \mu)$ containing $a$ and $b$, respectively. Hence, $(X, \mu)$ is $S p T_{2}$, as required.

In a similar way, one can prove the following results.

Proposition 10. Let $g:(X, \tau) \longrightarrow(Y, v)$ be a bijective supra preopen map. If $(X, \tau)$ is $T_{i}$, then $(Y, v)$ is $S p T_{i}$ for $i=0,1,2$.

Proposition 11. Let $g:(X, \tau) \longrightarrow(Y, \nu)$ be an injective supra pre $e^{\star}$ continuous map. If $(X, \tau)$ is $S p T_{i}$, then $(Y, \nu)$ is $S p T_{i}$ for $i=0,1,2$.

Proposition 12. Let $g:(X, \tau) \longrightarrow(Y, \nu)$ be a bijective supra pre $e^{\star}$-open map. If $(X, \tau)$ is $S p T_{i}$, then $(Y, v)$ is $S p T_{i}$ for $i=0,1,2$.

Proposition 13. Let $g:(X, \tau) \longrightarrow(Y, \nu)$ be a supra pre ${ }^{\star}$ homeomorphism map. Then, $(X, \tau)$ is $S p T_{i}$ iff $(Y, \nu)$ is $S p T_{i}$ for $i=0,1,2,3,4$.

Theorem 21. The finite product of $S p T_{i}$-spaces is $S p T_{i}$ for $i=0,1,2$.

Proof. We prove the theorem for two supra topological spaces $(X, \mu)$ and $(Y, \nu)$ in the case of $i=2$. One can prove the other cases similarly.

Let $(X \times Y, T)$ be the product supra space of $(X, \mu)$ and $(Y, \nu)$. Suppose that $\left(x_{1}, y_{1}\right) \neq\left(x_{2}, y_{2}\right)$. Then, either $x_{1} \neq x_{2}$ or $y_{1} \neq y_{2}$. Without loss of generality, suppose that $x_{1} \neq x_{2}$. Therefore, there exist two disjoint supra preopen subsets $U$ and $V$ of $(X, \mu)$ containing $x_{1}$ and $x_{2}$, respectively. It follows from Theorem 3 that $U \times Y$ and $V \times Y$ are two supra preopen subsets of $(X \times Y, T)$ containing $\left(x_{1}, y_{1}\right)$ and $\left(x_{2}, y_{2}\right)$, respectively, such that $(U \times Y) \tilde{\cap}(V \times Y)=\varnothing$. Hence, $(X \times$ $Y, T)$ is $S p T_{2}$.

Definition 20. Let $(X, \mu)$ and $(Y, v)$ be two supra topological spaces and $(X \times Y, T)$ be their product supra space such that $\mathscr{C}_{1}$ and $\mathscr{C}_{2}$ are the collections of all supra preopen subsets of $(X, \mu)$ and $(Y, \nu)$, respectively. Then, $\beta=\left\{G \times H: G \in \mathscr{C}_{1}\right.$ 
and $\left.H \in \mathscr{C}_{2}\right\}$ defines a basis for a supra topology $\mathscr{C}$ on $X \times Y$. We called $(X \times Y, \mathscr{C})$ a prefinite product supra space.

Lemma 2. Let $(X, \mu)$ and $(Y, \nu)$ be two supra topological spaces and $(X \times Y, \mathscr{C})$ be their preproduct supra space. If $E$ is a supra closed subset of $(X \times Y, \mathscr{C})$, then $E=\cap_{i \in I}$ $\left[\left(F_{i} \times Y\right) \cup\left(X \times H_{i}\right)\right]$, where $F_{i}$ and $H_{i}$ are supra preclosed subsets of $(X, \mu)$ and $(Y, \nu)$, respectively.

Theorem 22. The prefinite product of $S p T_{i}$-spaces is $S T_{i}$ for $i=0,1,2,3$.

Proof. We prove the theorem for two supra topological spaces $(X, \mu)$ and $(Y, \nu)$ in the case of $i=3$. One can prove the other cases similarly.

Let $(X \times Y, \mathscr{C})$ be the preproduct supra space of $(X, \mu)$ and $(Y, v)$. We first prove that $(X \times Y, \mathscr{C})$ is $S T_{1}$. Suppose that $\left(x_{1}, y_{1}\right) \neq\left(x_{2}, y_{2}\right)$. Then, either $x_{1} \neq x_{2}$ or $y_{1} \neq y_{2}$. Without loss of generality, suppose that $x_{1} \neq x_{2}$. Therefore, there exist two supra preopen subsets $U$ and $V$ of $(X, \mu)$ containing $x_{1}$ and $x_{2}$, respectively. According to Definition 20, $U \times Y$ and $V \times Y$ are two supra open subsets of $(X \times$ $Y, \mathscr{C})$ containing $\left(x_{1}, y_{1}\right)$ and $\left(x_{2}, y_{2}\right)$ such that $\left(x_{1}, y_{1}\right) \notin V \times Y$ and $\left(x_{2}, y_{2}\right) \notin U \times Y$. Hence, $(X \times Y, \mathscr{C})$ is $S T_{1}$. Second, we prove that $(X \times Y, \mathscr{C})$ is supra regular. Suppose that $(x, y) \in X \times Y$ and $E$ is a supra closed subset of $(X \times Y, \mathscr{C})$ such that $(x, y) \notin E=\cap_{i \in I}\left[\left(F_{i} \times Y\right) \cup\left(X \times H_{i}\right)\right]$, where $F_{i}$ and $H_{i}$ are supra preclosed subsets of $(X, \mu)$ and $(Y, \nu)$, respectively. Then, there exists $j \in I$ such that $(x, y) \notin\left[\left(F_{j} \times Y\right) \cup\left(X \times H_{j}\right)\right]$. This means that $x \notin F_{j}$ and $y \notin H_{j}$. Since $(X, \mu)$ and $(Y, \nu)$ are supra pre regular, then there exist disjoint supra preopen subsets $U$ and $V$ of $(X, \mu)$ containing $x$ and $F_{j}$, respectively, and there exist disjoint supra preopen subsets $M$ and $N$ of $(Y, \nu)$ containing $y$ and $H_{j}$, respectively. Therefore, $U \times M$ and $[(V \times Y) \cup(X \times N)]$ are two supra open subsets of $(X \times Y, \mathscr{C})$ containing $(x, y)$ and $\left[\left(F_{j} \times Y\right) \cup\left(X \times H_{j}\right)\right]$, respectively. Obviously, $E \subseteq\left[\left(F_{j} \times\right.\right.$ $\left.Y) \cup\left(X \times H_{j}\right)\right]$ and $(U \times M) \cap[(V \times Y) \cap(X \times N)]=\varnothing$. Thus, $(X \times Y, T)$ is supra regular. Hence, the proof is complete.

\section{Conclusion}

We began this work by correcting some results of [4]. Then, we have presented the concept of supra pre homeomorphism and investigated main properties. Also, we have introduced and studied the concepts of supra limits and supra boundary points with respect to preopen sets. Finally, we have defined the concepts of supra preregular, supra prenormal, and $S p T_{i}$-spaces $(i=0,1,2,3,4)$ and discussed their basic properties. From the concrete thoughts given in this work, more investigations can be carried out on the theoretical parts of these generalized ideas which are valuable by studying the following themes:

(1) Define weak types of supra preregular and supra prenormal spaces

(2) Study $S p T_{i}$-spaces for $1 / 2,21 / 2,31 / 2,5$
(3) Explore the concepts introduced herein using the classes of supra $\alpha$-open sets, supra semiopen sets, supra $b$-open sets, and supra $\beta$-open sets

(4) Investigate of the possibility of applying these concepts on information system, especially, separation axioms

\section{Data Availability}

No data were used to support this study.

\section{Conflicts of Interest}

The authors declare that they have no conflicts of interest.

\section{References}

[1] A. S. Mashhour, A. A. Allam, F. S. Mahmoud, and F. H. Kheder, "On supra topological spaces," Indian Journal of Pure and Applied Mathematics, vol. 14, pp. 502-510, 1983.

[2] T. Al-Shami, "Some results related to supra topological spaces," Journal of Advanced Studies in Topology, vol. 7, no. 4, pp. 283-294, 2016.

[3] R. Devi, S. Sampathkumar, and M. Caldas, "On $\alpha$-open sets and $s \alpha$-continuous maps," General Mathematics, vol. 16, pp. 77-84, 2008.

[4] O. R. Sayed, "Supra pre-open sets and supra pre-continuous on topological spaces," Series Mathematics and Information, vol. 20, pp. 79-88, 2010.

[5] O. R. Sayed and T. Noiri, "On supra $b$-open sets and supra $b$ continuity on topological spaces," European Journal of Pure and Applied Mathematics, vol. 3, pp. 295-302, 2010.

[6] S. Jafari and S. Tahiliani, "Supra $\beta$-open sets and supra $\beta$-continuity on topological spaces," Annales Universitatis Scientiarium Budapestinensis, vol. 56, pp. 1-9, 2013.

[7] M. E. El-Shafei, M. Abo-Elhamayel, and T. M. Al-Shami, "On supra $R$-open sets and some applications on topological spaces," Journal of Progressive Research in Mathematics, vol. 8, pp. 1237-1248, 2016.

[8] T. Al-Shami, "On supra semi open sets and some applications on topological spaces," Journal of Advanced Studies in Topology, vol. 8, no. 2, pp. 144-153, 2017.

[9] T. M. Al-Shami, "Utilizing supra $\alpha$-open sets to generate new types of supra compact and supra lindelöf spaces," Facta Universitatis, Series: Mathematics and Informatics, vol. 32, pp. 151-162, 2017.

[10] T. M. Al-Shami, "Supra semi-compactness via supra topological spaces," Journal of Taibah University for Science, vol. 12, no. 3, pp. 338-343, 2018.

[11] T. M. Al-Shami, B. A. Asaad, and M. A. El-Gayar, "Various types of supra pre-compact and supra pre-Lindelöf spaces," Missouri Journal of Mathematical Science, vol. 32, 2020.

[12] J. M. Mustafa, "Supra b-compact and supra $b$-Lindelof spaces," Journal of Mathematics and Applictions, vol. 36, pp. 79-83, 2013.

[13] R. M. Latif, "Supra-R-compactness and supra-R-connectedness," International Journal of Recent Trends in Engineering \& Research, vol. 4, no. 1, pp. 426-443, 2018.

[14] O. R. Sayed, "Supra $\beta$-connectedness on topological spaces," Proceedings of the Pakistan Academy of Sciences, vol. 49, pp. 19-23, 2012. 
[15] J. M. Mustafa and H. A. Qoqazeh, "Supra D-sets and associated separation axioms," International Journal of Pure and Applied Mathematics, vol. 80, pp. 657-663, 2012.

[16] T. M. Al-Shami and M. E. El-Shafei, "Two types of separation axioms on supra soft topological spaces," Demonstratio Mathematica, vol. 52, no. 1, pp. 147-165, 2019.

[17] A. M. Kozae, M. Shokry, and M. Zidan, "Supra topologies for digital plane," AASCIT Communications, vol. 3, pp. 1-10, 2016.

[18] S.-E. Han, "Hereditary properties of semi-separation axioms and their applications," Filomat, vol. 32, no. 13, pp. 46894700, 2018. 